\title{
Differences in $\mathrm{Ga}_{12^{-}}$and $\mathrm{Ga}_{13}$-mediated plasma membrane recruitment of p115-RhoGEF
}

\author{
Raja Bhattacharyya ${ }^{1,2,3}$, Jayashree Banerjee ${ }^{1}$, Kamel Khalili ${ }^{2}$, and Philip B. Wedegaertner ${ }^{1}$ \\ 1Department of Biochemistry and Molecular Biology, Thomas Jefferson University, Philadelphia, PA 19107 \\ 2Department of Neuroscience, Center for Neurovirology, Temple University School of Medicine, Philadephia, \\ PA 19122
}

\section{Abstract}

Regulator of G protein signaling domain-containing Rho guanine-nucleotide exchange factors (RGSRhoGEFs) directly link activated forms of the G12 family of heterotrimeric G protein $\alpha$ subunits to the small GTPase Rho. Stimulation of $\mathrm{G}_{12 / 13}$-coupled GPCRs or expression of constitutively activated forms of $\alpha_{12}$ and $\alpha_{13}$ has been shown to induce the translocation of the RGS-RhoGEF, p115-RhoGEF, from the cytoplasm to the plasma membrane (PM). However, little is known regarding the functional importance and mechanisms of this regulated PM recruitment, and thus PM recruitment of p115-RhoGEF is the focus of this report. A constitutively PM-localized mutant of p115-RhoGEF shows a much greater activity compared to wild type p115-RhoGEF in promoting Rho-dependent neurite retraction of NGF-differentiated PC12 cells, providing the first evidence that PM localization can activate p115-RhoGEF signaling. Next, we uncovered the unexpected finding that Rho is required for $\alpha_{13}$-induced PM translocation of p115-RhoGEF. However, inhibition of Rho did not prevent $\alpha_{12}$-induced PM translocation of p115-RhoGEF. Additional differences between $\alpha_{13}$ and $\alpha_{12}$ in promoting PM recruitment of p115-RhoGEF were revealed by analyzing RGS domain mutants of p115-RhoGEF. Activated $\alpha_{12}$ effectively recruits the isolated RGS domain of p115RhoGEF to the PM, whereas $\alpha_{13}$ only weakly does. On the other hand, $\alpha_{13}$ strongly recruits to the $\mathrm{PM}$ a p115-RhoGEF mutant containing amino acid substitutions in an acidic region at the $\mathrm{N}$-terminus of the RGS domain; however, $\alpha_{12}$ is unable to recruit this p115-RhoGEF mutant to the PM. These studies provide new insight into the function and mechanisms of $\alpha_{12 / 13}$-mediated PM recruitment of p115-RhoGEF.

\section{Keywords}

heterotrimeric G protein; Rho GTPase; guanine-nucleotide exchange factor; membrane translocation; regulator of $\mathrm{G}$ protein signaling (RGS)

\footnotetext{
Corresponding Author: Philip B. Wedegaertner, Ph. D., Department of Biochemistry and Molecular Biology, Thomas Jefferson University, 233 S. $10^{\text {th }}$ St., 839 BLSB, Philadelphia, PA 19107, tel: 215-503-3137, fax: 215-923-2117, e-mail: E-mail: P_Wedegaertner@mail.jci.tju.edu.

${ }^{3}$ Current Address: Neurobiology of Disease Laboratory, Genetics and Aging Research Unit, MassGeneral Institute for Neurodegenerative Disease (MIND) and Department of Neurology, Massachusetts General Hospital, Harvard Medical School, Charlestown, MA 02129

Publisher's Disclaimer: This is a PDF file of an unedited manuscript that has been accepted for publication. As a service to our customers we are providing this early version of the manuscript. The manuscript will undergo copyediting, typesetting, and review of the resulting proof before it is published in its final citable form. Please note that during the production process errors may be discovered which could affect the content, and all legal disclaimers that apply to the journal pertain.
} 


\section{INTRODUCTION}

Heterotrimeric $G$ proteins, composed of $\alpha, \beta$ and $\gamma$ subunits, function at the cytoplasmic surface of the plasma membrane to couple agonist-activated G protein-coupled receptors (GPCRs) to a wide variety of effectors and downstream signaling responses [1]. G proteins are classified into four families, Gs, Gi, Gq, and G12, based on $\alpha$ subunit sequence identity and signaling function. $\alpha_{12}$ and $\alpha_{13}$, the $\alpha$ subunits of the G12 family, share 65\% amino acid identity, and they share the ability to promote cellular proliferation, oncogenesis, cell migration, and cellular morphological changes. GPCRs that couple to $\alpha_{12}$ and $\alpha_{13}$ include LPA, thrombin, and thromboxane $\mathrm{A} 2$ receptors.

Many of the cellular responses promoted by $\alpha_{12}$ and $\alpha_{13}$ involve activation of the small GTPase Rho. $\alpha_{12}$ and $\alpha_{13}$ activate Rho by directly interacting with and activating a sub-family of Rho guanine-nucleotide exchange factors (RhoGEFs), which in turn catalyze GDP release and subsequent GTP binding to Rho. Although $\alpha_{12}$ and $\alpha_{13}$ have been shown to interact with a number of effectors [2], the RhoGEFs are the best characterized in terms of being demonstrated to clearly mediate important functions of $\alpha_{12}$ and $\alpha_{13}$.

More than 80 RhoGEFs have been identified [3], and most have the common feature of containing a tandem Dbl homology (DH) domain, which is responsible for guanine-nucleotide exchange activity, and pleckstrin homology (PH) domain, which appears to assist in catalysis and subcellular localization. Among these many RhoGEFs, only three RhoGEFs, termed p115RhoGEF, PDZ-RhoGEF and LARG, comprise the sub-family whose members are effectors for $\alpha_{12}$ and $\alpha_{13}$. The distinguishing feature of these three RhoGEFs is the presence of a regulator of G protein signaling (RGS) domain that mediates direct binding to activated $\alpha_{12}$ and $\alpha_{13}$. These RhoGEFs are thus often termed RGS-RhoGEFs.

The mechanisms of how activated $\alpha_{12}$ or $\alpha_{13}$ stimulate the Rho guanine-nucleotide exchange activity of these RGS-RhoGEFs remain to be fully elucidated [4]. What is clear is that activated $\alpha_{12 / 13}$ binding to the RGS domain is essential. However, the simplest model whereby $\alpha_{12 / 13}$ binding to the RGS domain is sufficient for RGS-RhoGEF activation by relieving autoinhibition does not adequately account for experimental results [4]. An alternative model suggests that $\alpha_{12 / 13}$ also interacts with a second surface on the DH-PH domain [4-6], and this interaction is necessary for stimulation of guanine-nucleotide exchange activity. Moreover, there is evidence that in some cases covalent modification of the RGS-RhoGEF by phosphorylation is required for activation by $\alpha_{12}$ or $\alpha_{13}$ [7,8]. To complicate matters further, in vitro reconstitution assays have revealed differences in the ability of $\alpha_{12}$ versus $\alpha_{13}$ to activate RGS-RhoGEFs. For example, $\alpha_{13}$, but not $\alpha_{12}$, can stimulate p115-RhoGEF and LARG; phosphorylation of LARG allows stimulation by $\alpha_{12}$, but how or if $\alpha_{12}$ stimulates p115RhoGEF has not been resolved.

An additional mechanism that has been proposed to contribute to regulation of RGS-RhoGEFs by $\alpha_{12 / 13}$ is plasma membrane (PM) translocation. Although PDZ-RhoGEF is localized to the cortical actin cytoskeleton, both p115-RhoGEF and LARG display predominant localization in the cytoplasm. Moreover, p115-RhoGEF and LARG have been observed to re-localize from the cytoplasm to the PM upon expression of constitutively active $\alpha_{12}$ or $\alpha_{13}$ and agonist stimulation of endogenous or overexpressed G12/13-coupled GPCRs [4,9-15]. The mechanisms of the $\alpha_{12 / 13}$-mediated PM translocation of RGS-RhoGEFs are not fully understood. Recruitment to the PM requires the RGS domain, but not an N-terminal acidic region of the RGS domain that is required for high affinity binding to $\alpha_{12 / 13}$ [4,13]. In addition, the PH domain of p115-RhoGEF is required for its PM recruitment [12], further suggesting that multiple factors contribute to productive PM recruitment of RGS-RhoGEFs. Furthermore, although it is clear that RGS-RhoGEFs can be recruited from the cytoplasm to the PM in 
response to $\alpha_{12 / 13}$ activation, it has not been demonstrated that such PM recruitment is indeed important for RGS-RhoGEF function.

In this report, we provide new insight into the function and mechanism of PM recruitment of p115-RhoGEF. First, assaying Rho-dependent neurite retraction in PC12 cells we show that expression of a PM-targeted p115-RhoGEF induces neurite retraction, whereas cytoplasmic wild type p115-RhoGEF is mostly unable to effect neurite retraction by itself. These results are the first clear evidence that PM recruitment is sufficient to activate signaling. Next, we uncovered the unexpected finding that Rho, which is presumably downstream of p115RhoGEF in the signaling cascade, is required for $\alpha_{13}$-induced PM translocation of p115RhoGEF. Lastly, we demonstrated key differences between $\alpha_{13}$ and $\alpha_{12}$ in PM recruitment of p115-RhoGEF. Whereas $\alpha_{13}$ displays a novel requirement for Rho in PM recruitment of p115RhoGEF, $\alpha_{12}$ does not. Activated $\alpha_{12}$ effectively recruits the isolated RGS domain of p115RhoGEF to the PM, whereas $\alpha_{13}$ only weakly does. On the other hand, $\alpha_{13}$ strongly recruits to the PM a p115-RhoGEF mutant containing amino acid substitutions in an acidic region at the $\mathrm{N}$-terminus of the RGS domain, but $\alpha_{12}$ is unable to recruit this p115-RhoGEF mutant to the PM.

\section{MATERIALS AND METHODS}

\subsection{Plasmids and expression vectors}

The N-terminal myc epitope (MEQKLISEED)-tagged pcDNA3-myc-p115-RhoGEF, the hemagglutinin (HA) epitope (DVPDYA)-tagged pcDNA3 $\mathrm{G}_{13} \mathrm{QL}\left(\alpha_{13} \mathrm{QL}\right)$, untagged $\mathrm{G} \alpha_{12} \mathrm{QL}\left(\alpha_{12} \mathrm{QL}\right)$ in pcDNAI, the C-terminal green fluorescence protein (GFP)-tagged p115RhoGEF, and the expression vector for C3-transferase (C3), were described previously [12, 14]. The expression plasmid carrying HA epitope-tagged activated Rho cDNA (RhoV14) was obtained from P. Tsichlis (Kimmel Cancer Institute, Philadelphia). The Stratagene QuickChange Site-directed Mutagenesis Kit was used to replace $\mathrm{Glu}^{27}, \mathrm{Glu}^{29}$ and $\mathrm{Asp}^{30}$ of p115-RhoGEF with Ala to generate p115-RhoGEF(EED).

\subsection{Cell culture and transfection}

PC12 cells were plated on collagen IV (Sigma, MO) coated plates in Dulbecco's modified Eagle's medium (DMEM) containing 10\% fetal bovine serum (FBS) and 5\% horse serum supplemented with penicillin and streptomycin. PC12 cells were transfected using Lipofectamine 2000 (Invitrogen) as per the manufacturer's protocol. $24 \mathrm{~h}$ after transfection neurite extension was induced by exposing the cells to serum free medium containing $100 \mathrm{ng} /$ $\mathrm{ml}$ nerve growth factor (NGF) for an additional $16 \mathrm{~h}$. Transfection of HEK293 cells were performed as described before [12].

\section{3 siRNA transfection}

The Rho sense and anti-sense siRNA oligonucleotides 5' (GAAGUCAAGCAUUUCUGUC) dTdT 3' and 5' (GACAGAAAUGCUUGACUUC)dTdT 3', respectively, were purchased from Dharmacon Research, Inc., CO. A pair of scrambled siRNA was obtained from Jeff Benovic (Thomas Jefferson University, Philadelphia) as a control. Transfection of siRNA was achieved by using Oligofectamine (Invitrogen) as per manufacturer's protocol. Complete silencing of Rho was achieved by transfecting the cells twice after $24 \mathrm{~h}$ intervals with the siRNA.

\subsection{Immunofluorescence Assay}

Cells were grown on collagen IV coated chamber slides (for PC12 cells) or uncoated cover slips (for HEK293 cells), and were transfected with appropriate plasmids. The cells were subjected to immunofluorescence microscopy as done earlier [12], after probing with anti-myc 
9E10 (Covance or Novagen), anti-HA 12CA5 (Covance or Roche) and/or anti- $\alpha_{12}$ polyclonal antibody (Santa Cruz Biotech., CA). Goat anti-mouse antibodies conjugated with FITC (Vector Laboratories, CA), Alexa 488 or Alexa 594 (Invitrogen), and goat anti-rabbit antibodies conjugated with Texas Red (Vector Laboratories, CA) or Alexa 594 (Invitrogen) were used in our immunofluorescent labeling. For actin staining, the chamber slides were prepared for fluorescence microscopy as mentioned above, with the exception that a 1:50 dilution of Alexa594 conjugated phalliodin (Molecular Probes) was included during the secondary antibody incubation.

\subsection{Subcellular fractionation}

HEK 293 cells were plated at $\sim 2 \times 10^{6}$ cells in $6 \mathrm{~cm}$ plates, grown for $24 \mathrm{~h}$ and transfected with $3 \mu \mathrm{g}$ of total plasmids. Cells were fractionated into membrane bound P-fractions and cytoplasmic S-fractions as done before [12]. We used anti-myc and anti-HA antibodies to detect myc- and HA epitope-tagged proteins, respectively, on immunoblots. Expression of endogenous Rho was detected by using anti-Rho antibody (Upstate, CA) at 1:2000 dilution.

\subsection{Cell Morphology}

PC12 cells were transfected with either GFP vector alone or with other expression plasmids. $24 \mathrm{~h}$ later the cells were stimulated with $100 \mathrm{ng} / \mathrm{ml}$ NGF for an additional $16 \mathrm{~h}$, before fixing. Cells were subjected to immunofluorescence assay. Two types of morphologies were detected, i) neurite-bearing cells or ii) rounded cells. The percent $(\%)$ cell rounding by overexpressed proteins was calculated and plotted as described elsewhere [16], with some modification: \% cell rounding $=$ (the number of round fluorescent labeled cells $/$ total number of fluorescent cells counted) $\times 100$. More than 100 cells were counted for five random fields in at least 3 independent experiments.

\subsection{Co-immunoprecipitation}

Co-immunoprecipitations of p115-RhoGEF with $\alpha_{13} \mathrm{QL}$ and $\alpha_{12} \mathrm{QL}$ were performed as described previously [12].

\subsection{TUNEL Assay}

Cell death was detected by TUNEL assay with In Situ Death Detection kit (Roche, Indianapolis, IN) as described by Wang et. al.[21]. 24h post-tranfected PC12 cells were treated with $100 \mathrm{ng} /$ $\mathrm{ml}$ NGF for additional $16 \mathrm{~h}$ prior to TUNEL assay. Cell death was evaluated by detecting DNA strand break under a fluorescent microscope equipped with a set of excitation-emmission filters for fluorescein. Fluorescein positive cells were counted and compared among cells expressing GFP, p115-RhoGEF, $\alpha_{13} \mathrm{QL}$, or their mutants. As a positive control, PC 12 cells were treated with $50 \mathrm{ng} / \mathrm{ml}$ TNF $\alpha$ (BD Biosciences, Palo Alto, CA) for 40 and $96 \mathrm{hrs}$. In each case cells were subjected to NGF treatment after $24 \mathrm{~h}$ TNF $\alpha$ treatment to mimic transfection experiments.

\section{RESULTS}

\subsection{PM association of p115-RhoGEF induces Rho-dependent neurite retraction in PC12 cells}

We and others have demonstrated that constitutively activated forms of $\alpha_{12}$ and $\alpha_{13}$ and stimulation of $\mathrm{G}_{12 / 13}$-coupled GPCRs induces the translocation of p115-RhoGEF from the cytoplasm to the plasma membrane (PM) [4,9,11-15]. However, no studies have yet demonstrated that PM recruitment plays a role in activation of p115-RhoGEF-mediated signaling. Here, we examined the effect of PM association of p115-RhoGEF on neurite retraction of NGF-differentiated PC12 cells, a well characterized model system in which neurite retraction induced by $\alpha_{12 / 13}$ and $\mathrm{G}_{12 / 13}$-coupled GPCRs depends on activation of Rho [17-21]. In these experiments, PC12 cells were transiently transfected with a GFP expression 
plasmid, in the presence or absence of additional expression vectors, and then allowed to differentiate in the presence of NGF. GFP expressing cells were scored for the presence of neurite extensions or for showing round morphology lacking neurite extensions (Figure 1). In the absence of NGF, the majority of GFP-expressing PC12 cells $(57.40 \pm 3.0 \%, \mathrm{p}<0.0001)$ displayed a rounded morphology lacking neurites (Fig. 1B), while in the presence of NGF $80-90 \%$ of the cells contained clear neurite extensions (Fig. 1A.a and Fig. 1B). When cells were also transfected with a vector for constitutively active $\alpha_{13} \mathrm{QL}$, a majority of GFPexpressing PC12 cells displayed a dramatic inhibition of NGF-stimulated neurite extension (Fig. 1A.c and Fig. 1B), consistent with previous reports of $\alpha_{13}$ QL-stimulated neurite retraction [21]. As a control, a non-palmitoylated form of $\alpha_{13}$ QL, termed $\alpha_{13}$ QL-CCSS [14], had no effect on NGF-induced neurites, in agreement with our previous report showing that $\alpha_{13}$ QL-CCSS is in an activated conformation but unable to activate Rho-dependent signaling [14] (Fig 1A.e). The critical dependence on Rho for neurite retraction was demonstrated by expression of C3transferase (C3), a toxin that specifically inhibits Rho by catalyzing its ADP-ribosylation [22]. In the absence of NGF treatment, C3-mediated inhibition of Rho dramatically induced neurites (Fig. 1B), and in the presence of NGF treatment these cells showed longer and more branched neurites compared to cells expressing only GFP (Fig 1A, compare g and a). Thus, the above described experiments confirmed the suitability of the PC12 system for examining regulators of Rho.

Next, p115-RhoGEF was expressed along with GFP in PC12 cells. Only $15.81 \pm 1.7 \%$ $(\mathrm{p}=0.0008)$ cells expressing $\mathrm{p} 115$-RhoGEF displayed the ability to induce neutrite retraction in NGF-differentiated cells (Fig. 1A.i and Fig. 1B). However, when $\alpha_{13} \mathrm{QL}$ and p115-RhoGEF were expressed together, neurite retraction (i.e., cell rounding) was strongly observed in 91 $\pm 1.4 \%(\mathrm{p}=0.0007)$ NGF-treated cells (Fig. 1A.k and Fig. 1B). Although $\alpha_{13} \mathrm{QL}$ recruits $\mathrm{p} 115$ RhoGEF to the PM in PC12 cells, these data cannot indicate that PM recruitment of p115RhoGEF is functionally important. To test this, we generated a mutant of p115-RhoGEF that is constitutively targeted to the PM. This was accomplished by fusing the C-terminal 20 amino acids from $\mathrm{H}$-Ras to the C-terminus of p115-RhoGEF, to generate p115-RhoGEF-CAAX (Fig. $\mathrm{S} 1$ ). A number of studies have demonstrated that this region of $\mathrm{H}$-Ras undergoes farnesylation and palmitoylation, and is sufficient to target heterologous proteins to the PM [23, 24]. In contrast to wild type cytoplasmic p115-RhoGEF, PM-localized p115-RhoGEF-CAAX strongly induced neurite retraction (i.e. cell rounding) in $87 \pm 2.2 \%$ ( $\mathrm{p}=0.0001$ ) of NGF-treated cells. (Fig. 1A.m and Fig. 1B). This result provides the first evidence that PM localization of p115-RhoGEF can activate Rho-dependent signaling. When p115-RhoGEF-CAAX contained a mutation in the PH domain that inhibits Rho-dependent signaling [12], NGF-stimulated neurite extensions were unaffected (Fig. 1A.o and Fig. 1B); cells showed long neurites, consistent with PM-associated p115-RhoGEF-CAAX inducing neurite retraction via a Rhodependent pathway. Lastly, when we evaluated apoptosis by TUNEL assay, as described in a previous report [25], we observed no differences among the TUNEL positive cells expressing p115-RhoGEF or $\alpha_{13}$ QL or their mutants compared to cells expressing control GFP (Fig. 1D) $40 \mathrm{~h}$ post-transfection, suggesting that the observed effects on neutrite retraction and cell rounding in Figure 1 are not due to cell death. As a positive control for the TUNEL assay, we treated PC12 cells with tumor necrosis factor $\alpha(\mathrm{TNF} \alpha)$ that is known to induce cell death [26]. $40 \mathrm{~h}$ treatment of $50 \mathrm{ng} / \mathrm{ml} \mathrm{TNF} \alpha$ produced $~ 10 \%$ TUNEL positive cells, which reached almost 50\% after $96 \mathrm{~h}$ treatment (Fig. 1D). All immunofluorescence assays in Figure 1 were performed $40 \mathrm{~h}$ post-transfection. In summary, neurite retraction and cell rounding of NGFdifferentiated PC12 cells provides a model system for studying Rho-dependent signaling. The key result using this system is that expression of p115-RhoGEF only weakly activates this signaling pathway, but simply PM-targeting of p115-RhoGEF (i.e., p115-RhoGEF-CAAX) is able to strongly enhance its ability to induce neurite retraction and cell rounding. 


\subsection{Inhibition of Rho abrogates $\alpha_{13}$-mediated PM translocation of p115-RhoGEF}

During the course of these studies, we noted that p115-RhoGEF failed to be recruited to the PM by constitutively active $\alpha_{13}$ QL when Rho was inhibited by C3. Rho is downstream of and directly activated by p115-RhoGEF, and it is therefore surprising and unexpected that inhibition of Rho would affect PM recruitment of p115-RhoGEF. Thus, we proceeded to examine the requirement for Rho in $\alpha_{13}$ QL-mediated PM recruitment of p115-RhoGEF.

In PC12 cells, expressed p115-RhoGEF is distributed throughout the cytoplasm (Fig. 2a), and co-expression of $\alpha_{13} \mathrm{QL}$ strongly recruits p115-RhoGEF to the PM as shown by the intense staining at the cell periphery (Fig. 2d). However, when C3 is expressed together with $\alpha_{13} \mathrm{QL}$ and p115-RhoGEF, p115-RhoGEF remains in the cytoplasm and is not detected at the PM (Fig. $2 \mathrm{~g}$ and $2 \mathrm{j}$ ). A similar cytoplasmic localization of $\mathrm{p} 115-\mathrm{RhoGEF}$ is observed when $\mathrm{C} 3$ is coexpressed with p115-RhoGEF in the absence of $\alpha_{13} \mathrm{QL}$ (Fig $2 \mathrm{~m}$ ). C3 did not affect the localization of $\alpha_{13} \mathrm{QL} ; \alpha_{13} \mathrm{QL}$ displayed a subcellular distribution of both PM and cytoplasmic staining in the absence (Fig 2e) or presence of $\mathrm{C} 3$ (Fig $2 \mathrm{~h}$ and $2 \mathrm{k}$ ). The functional inhibition of Rho by $\mathrm{C} 3$ is evident by the observation that $\mathrm{C} 3$ expression induces neurite extensions (Fig. $2 \mathrm{~g}-\mathrm{o}$ ). In this set of experiments, a GFP-tagged $\mathrm{p} 115$-RhoGEF was utilized; $\mathrm{N}$-terminally myctagged and C-terminally GFP-tagged forms of p115-RhoGEF are both effectively recruited to the PM by $\alpha_{13}$ QL in PC12 and HEK293 cells. Because C3 prevents the activation of Rho, these experiments suggest a novel requirement of activation of Rho in facilitating PM recruitment of p115-RhoGEF.

To further characterize the requirement for Rho activation, we examined whether Rho activation was sufficient for PM recruitment of p115-RhoGEF and whether downstream Rho signaling to the actin cytoskeleton was necessary. When the constitutively active RhoA V14 mutant was co-expressed in PC12 cells with p115-RhoGEF, p115-RhoGEF remained in the cytoplasm (Fig. 2p) and did not translocate to the PM. This result indicates that, whereas active Rho is necessary for p115-RhoGEF PM recruitment, it is not sufficient. One of the important signaling pathways mediated by Rho leads to increases in cellular F-actin, and consequently inhibition of Rho with $\mathrm{C} 3$ leads to loss of F-actin structures [27]. To examine a role for an intact actin cytoskeleton in $\alpha_{13}$ QL-promoted PM recruitment of p115-RhoGEF, cells were treated with latrunculin B which causes the rapid shift of a cell's F-actin to G-actin and thereby disrupts the actin cytoskeleton. In latrunculin B treated cells, p115-RhoGEF retained the ability to be recruited to the PM in response to co-expression of $\alpha_{13} \mathrm{QL}$ (data not shown). Thus, the inhibition by $\mathrm{C} 3$ of $\alpha_{13} \mathrm{QL}$-promoted PM recruitment of p115-RhoGEF together with the latrunculin B experiment suggests that Rho facilitates PM recruitment of p115-RhoGEF through a mechanism that does not involve regulated changes in the actin cytoskeleton.

A requirement for Rho in PM recruitment of p115-RhoGEF was confirmed through biochemical fractionation and the use of siRNA depletion of Rho. In these experiments, we utilized HEK293 cells because a higher transfection efficiency, compared to PC12 cells, was required to detect sufficient amounts of expressed protein in biochemical fractionation experiments. First, we confirmed by immunofluorescence that $\mathrm{C} 3$ inhibition of Rho showed the same effect on p115-RhoGEF PM recruitment in HEK293 cells as it did in PC12 cells. Indeed, $\alpha_{13}$ QL induced PM localization of p115-RhoGEF in HEK293 cells, as reported previously [12-14], but co-expression of C3 prevented PM recruitment of p115-RhoGEF (Fig. $3 \mathrm{~A})$. Thus, we proceeded to biochemical fractionation experiments. Cells were lysed in a hypotonic buffer, and high-speed centrifugation separated the cell lysate into membrane-rich particulate $(\mathrm{P})$ and cytoplasm-rich soluble (S) fractions. When expressed alone, p115-RhoGEF was detected almost exclusively in the soluble fraction (Fig 3B, lanes 1S and 1P), but p115RhoGEF strongly partitioned into the $\mathrm{P}$ fraction (approximately 50:50, S:P) when co-expressed with $\alpha_{13} \mathrm{QL}$ (Fig 3B, lanes $2 \mathrm{~S}$ and 2P). However, co-expression of C3-transferase with $\alpha_{13} \mathrm{QL}$ and p115-RhoGEF, abrogated the partitioning of p115-RhoGEF into the P fraction (Fig 3B, 
lanes $3 \mathrm{~S}$ and 3P), consistent with fluorescence microscopy results (Fig. 2 and 3A). As a complementary technique to C3-mediated inhibition of Rho, siRNA was used to deplete RhoA protein in HEK293 cells. Using a previously reported siRNA [28], RhoA was efficiently depleted (Fig. 3B, right panel, lane 4). In these RhoA-depleted cells, p115-RhoGEF failed to be recruited to the $\mathrm{P}$ fraction when $\alpha_{13} \mathrm{QL}$ was co-expressed (Fig. 3B, lanes $4 \mathrm{~S}$ and $4 \mathrm{P}$ ). A control siRNA had no effect; $\mathrm{p} 115-\mathrm{RhoGEF}$ still partitioned into the $\mathrm{P}$ fraction when coexpressed with $\alpha_{13} \mathrm{QL}$ (Fig. 3B, lanes 5S and 5P). Taken together, the fluorescence microscopy and biochemical fractionation, using $\mathrm{C} 3$-mediated inhibition and siRNA-mediated depletion of Rho, indicate a novel role for Rho in regulated PM translocation of p115-RhoGEF.

\section{3 $\alpha_{12}$-mediated PM translocation of p115-RhoGEF does not require Rho}

Since both $\alpha_{12}$ and $\alpha_{13}$ promote PM translocation of p115-RhoGEF [12], we tested whether inhibition of Rho also prevented $\alpha_{12}$ QL-mediated PM recruitment of p115-RhoGEF. Surprisingly, expression of C3-transferase did not prevent $\alpha_{12}$ QL-mediated PM recruitment of p115-RhoGEF, in contrast to what was observed for $\alpha_{13} \mathrm{QL}-$ mediated PM recruitment of p115RhoGEF. In both PC12 cells (not shown) and HEK293 cells, co-expression of $\alpha_{12}$ QL with p115-RhoGEF promoted strong PM localization of p115-RhoGEF regardless of whether C3 transferase was also expressed (Fig. 4A). Biochemical fractionation confirmed the lack of effect of C3. Expression of $\alpha_{12}$ QL promoted a strong shift of p115-RhoGEF to the P fraction (Fig. 4B, lanes $2 \mathrm{~S}$ and $2 \mathrm{P}$ ) and this recruitment to the $\mathrm{P}$ fraction was not perturbed by expression of $\mathrm{C} 3$ (Fig. 4B, lanes 3S and 3P). Thus, these results provide the first indication that there are mechanistic differences in the PM recruitment of p115-RhoGEF that is mediated by $\alpha_{12}$ versus $\alpha_{13}$.

\subsection{RGS domain mutants of p115-RhoGEF reveal differences in $\alpha_{12}$ and $\alpha_{13}$-induced PM recruitment}

To further explore differences in $\alpha_{12}$-mediated versus $\alpha_{13}$-mediated PM recruitment of $\mathrm{p} 115$ RhoGEF, we analyzed previously described [12] p115-RhoGEF mutants. A p115-RhoGEF mutant lacking the N-terminal RGS domain, (246-912)p115-RhoGEF remained cytoplasmic when co-expressed with either $\alpha_{13} \mathrm{QL}$ [12] or $\alpha_{12} \mathrm{QL}$ (not shown), confirming that the RGS domain is essential, presumably due to its ability to interact with $\alpha_{13} \mathrm{QL}$ and $\alpha_{12} \mathrm{QL}$. However, a clear difference between $\alpha_{13} \mathrm{QL}$ and $\alpha_{12} \mathrm{QL}$ was observed when PM recruitment of the isolated $\mathrm{N}$-terminal RGS domain of p115-RhoGEF was assayed. (1-246)p115-RhoGEF coimmunoprecipitates with both $\alpha_{12} \mathrm{QL}$ and $\alpha_{13} \mathrm{QL}$ (Fig. 5A, lane 4; and 5B, lane 4), consistent with previous reports showing interaction of the RGS domain of p115-RhoGEF with both $\alpha_{13}$ and $\alpha_{12}$ in an activation-dependent manner [6,29,30]. As described previously [12], (1-246) p115-RhoGEF is only weakly recruited from the cytoplasm to the PM when co-expressed with $\alpha_{13}$ QL (Fig. 6A) and shows little or no shift to the P fraction in biochemical fractionation assays (Fig. 6C). Thus, the RGS domain is not sufficient for $\alpha_{13}$ QL-mediated PM recruitment. On the other hand, we now find that $\alpha_{12} \mathrm{QL}$, compared to $\alpha_{13} \mathrm{QL}$, induces a more robust recruitment of (1-246)p115-RhoGEF to the PM (Fig. 6A) and promotes a substantial shift of (1-246)p115RhoGEF to the $\mathrm{P}$ fraction (Fig. 6B). In other words, the N-terminal RGS domain of p115RhoGEF is both necessary and sufficient for $\alpha_{12}$ QL-promoted PM recruitment.

An analysis of regulated PM recruitment of a RGS domain mutant of p115-RhoGEF reveals another difference between $\alpha_{13}$ and $\alpha_{12}$. The RGS domain of p115-RhoGEF consists not only of the conserved RGS core (amino acids 44-233) but also a short N-terminal extension marked by a stretch of acidic residues (amino acids 27-34); a recent crystal structure indicates that $\alpha_{13}$ interacts with a surface in the RGS core and with the $\mathrm{N}$-terminal acid region [31]. Moreover, mutagenesis studies have indicated that the $\mathrm{N}$-terminal acidic residues are required for high affinity interaction between $\alpha_{13}$ and p115-RhoGEF $[4,13,31,32]$. We previously reported the surprising result that whereas activated $\alpha_{13}$ failed to co-immunoprecipitate with an acidic 
region mutant of full-length $\mathrm{p} 115-\mathrm{RhoGEF}$, the acidic region mutant of $\mathrm{p} 115-\mathrm{RhoGEF}$ retained the ability to be strongly recruited to membranes by $\alpha_{13} \mathrm{QL}$ [13]. This was interpreted as a loss of high affinity interaction between $\alpha_{13}$ and p115-RhoGEF due to loss of the critical acidic residues and thus consequent loss of stable interaction in a co-immunoprecipitation; however, weaker interactions between $\alpha_{13}$ and the core RGS domain of p115-RhoGEF would be sufficient to recruit the mutant p115-RhoGEF to the PM where additional factors, such as $\alpha_{13}$ binding to additional domains (e.g., the DH domain) of p115-RhoGEF, interactions of the PH domain with membrane lipids, and likely a Rho-dependent factor, as described herein, would allow for stable membrane binding of p115-RhoGEF [4,13]. Consistent with our previous report, we show that an acidic mutant, termed p115-RhoGEF(EED) in which glutamic acids 27 and 29 and aspartic acid 30 are all changed to alanines, does not efficiently coimmunoprecipitate with $\alpha_{13} \mathrm{QL}$ (Fig. 5B, lane 3) but is strongly recruited to the PM (Fig. 6A) and to the $\mathrm{P}$ fraction in biochemical fractionation assays (Fig. 6C). Like $\alpha_{13} \mathrm{QL}, \alpha_{12} \mathrm{QL}$ is severely impaired in interacting with p115-RhoGEF(EED) as evidenced by poor coimmunoprecipitation (Fig. 5A, lane 3), suggesting that $\alpha_{12}$ also requires the $\mathrm{N}$-terminal acidic region of the RGS domain for strong interaction with p115-RhoGEF. However, in contrast to $\alpha_{13} \mathrm{QL}, \alpha_{12} \mathrm{QL}$ expression failed to recruit p115-RhoGEF(EED) to the PM (Fig. 6A) and failed to cause a shift of p115-RhoGEF(EED) to the P fraction (Fig. 6B). Taken together results in Figure 6 demonstrate fundamental differences in how $\alpha_{13}$ and $\alpha_{12}$ promote the translocation of p115-RhoGEF to the PM.

\section{DISCUSSION}

Regulated and reversible translocation of proteins from one subcellular location to another is a common feature of signal transduction pathways. One example of protein translocation is the activation-dependent recruitment of specific proteins from the cytoplasm to the PM in order to form localized signaling complexes. The results described herein provide several novel insights into the function and mechanism of $\alpha_{12 / 13}$-mediated PM recruitment of p115-RhoGEF.

This report provides the first evidence that PM localization is actually important for Rhodependent signaling functions of p115-RhoGEF. Although it has been thought that PM recruitment might be important for $\mathrm{p} 115$-RhoGEF function, results to support this have been lacking previously. We employed a well-characterized cell-based assay for Rho function in which neurite retraction of NGF-differentiated PC12 cells has been shown to be induced by $\alpha_{12 / 13}$ and $G_{12 / 13}$-coupled GPCRs in a Rho-dependent manner [17-21]. To test for a role for PM localization in p115-RhoGEF function, expression of wild type p115-RhoGEF was compared with a constitutively PM-localized p115-RhoGEF-CAAX, in which the C-terminal 20 amino acids of $\mathrm{H}$-Ras was appended to the C-terminus of p115-RhoGEF to provide a strong PM targeting signaling. Whereas expression of wild type p115-RhoGEF showed very weak ability to reverse the NGF-differentiated phenotype of neurite extensions in PC12 cells, p115RhoGEF-CAAX completely reversed the NGF-promoted neurite extensions. This key result indicates that, at least for some Rho-dependent signaling functions, PM recruitment of an RGSRhoGEF is sufficient to activate a signaling pathway. Importantly, PM recruitment of expressed or endogenous p115-RhoGEF has been observed in response to extracellular ligand activation of several $\mathrm{G}_{12 / 13}$-coupled GPCRs, such as LPA, thromboxane A2, sphingosine-1phosphate and thrombin receptors $[9,12,15]$, indicating that PM recruitment of $\mathrm{p} 115-\mathrm{RhoGEF}$ occurs under acute GPCR and G protein activation. Although it is likely that PM recruitment is not the only way in which $\alpha_{12 / 13}$ activates p115-RhoGEF, the results presented here, combined with previous studies [14], confirm the importance of regulated PM localization for p115-RhoGEF function.

The studies in this report also describe an unexpected role for Rho in the $\alpha_{13}$-promoted PM recruitment of p115-RhoGEF. Immunofluorescence microscopy and biochemical fractionation 
(Fig. 2 and 3) strikingly show that inhibition of Rho by the toxin $\mathrm{C} 3$ transferase or that siRNA depletion of Rho completely abrogates the $\alpha_{13}$ QL-mediated PM recruitment of p115-RhoGEF. This is surprising because Rho is activated by p115-RhoGEF and thus is downstream of the $\alpha_{13} / \mathrm{p} 115-$ RhoGEF interaction. The mechanism of the requirement for Rho is unclear and is the subject of future studies. Loss of $\alpha_{13}$-mediated PM recruitment of p115-RhoGEF upon Rho inhibition or depletion does not appear to be an indirect effect of disruption of the cell's actin cytoskeleton since depolymerization of F-actin by treatment with latrunculin B did not prevent $\alpha_{13}$-dependent PM translocation of p115-RhoGEF (not shown). Moreover, the presence of overexpressed, constitutively active Rho is not sufficient to induce PM localization of p115RhoGEF (Figure 2), suggesting that the requirement for Rho is one of several factors that can influence the important PM recruitment of p115-RhoGEF. We can speculate on several potential mechanisms of how Rho could promote the regulated PM localization of p115RhoGEF. Rho may be required to help tether p115-RhoGEF to the PM through direct binding or indirectly via additional scaffolding proteins, and this function could be promoted in a feedback manner. Alternatively, activation of Rho, either directly by p115-RhoGEF or in a parallel pathway initiated by active $\alpha_{13}$, could activate a positive feedback signaling response to enhance $\mathrm{p} 115$-RhoGEF PM recruitment. Interestingly, a recent report uncovered a positive feedback response in the activation of LARG [33]. The Rho effector Dial was shown to bind LARG and enhance LARG's in vitro guanine-nucleotide exchange activity, thus providing a positive feedback loop. It will be important to test whether Dia1 plays a role in $\alpha_{13}$-promoted PM recruitment of LARG and p115-RhoGEF.

The results in this report also describe novel mechanistic differences in $\alpha_{13}$-mediated versus $\alpha_{12}$-mediated PM recruitment of p115-RhoGEF. First, $\alpha_{12}$-mediated PM recruitment of p115RhoGEF was not prevented by $\mathrm{C} 3$ transferase inhibition of Rho (Fig. 4). This result is in striking contrast to the requirement for Rho in $\alpha_{13}$-mediated PM recruitement of p115-RhoGEF. Second, our studies revealed p115-RhoGEF RGS domain-based differences in $\alpha_{13}$-mediated versus $\alpha_{12}$-mediated PM recruitment. Whereas $\alpha_{13} \mathrm{QL}$ shows little or no ability to recruit to the PM the isolated N-terminal RGS domain (aa 1-246) of p115-RhoGEF (Fig. 6A and 6B) [12], $\alpha_{12} \mathrm{QL}$ strongly recruited the isolated RGS domain to the PM and to the membrane fraction, as assayed by immunofluorescence microscopy and biochemical fractionation (Fig. 6A and 6C). This result suggests that $\alpha_{12} \mathrm{QL}$ compared to $\alpha_{13} \mathrm{QL}$ interacts more strongly with p115-RhoGEF's RGS domain. However, in co-immunoprecipitation or pull-down experiments, the RGS domain of p115-RhoGEF appears to interact equally well with activated $\alpha_{12}$ and $\alpha_{13}$ (Fig. 5) [6]. Another set of experiments examined the ability of $\alpha_{12}$ QL versus $\alpha_{13} \mathrm{QL}$ to recruit to the PM a mutant p115-RhoGEF in which an N-terminal acidic region of the RGS domain (aa 27-32) was disrupted by substitution with alanines. This $\mathrm{N}$-terminal acidic stretch provides a second distinct site of RGS domain interaction with $\alpha_{13}$ [31,32]; the other contact surface utilizes multiple loops within amino acids 44-233 of the RGS domain. The Nterminal acidic region is necessary for the GAP activity of the RGS domain towards $\alpha_{13}$ [31, $32]$ and for high-affinity binding of p115-RhoGEF to $\alpha_{13}[4,13,32]$. However, mutational disruption of the acidic region does not inhibit the ability of $\alpha_{13}$ to stimulate the Rho exchange activity of p115-RhoGEF [32], and, importantly for the studies herein, does not prevent the $\alpha_{13}$-mediated PM recruitment of p115-RhoGEF [13]. In agreement with other reports, we show that $\alpha_{13}$ QL poorly interacts, via co-immunoprecipitation (Fig. 5B), with an $\mathrm{N}$-terminal acidic region EED mutant of p115-RhoGEF; additionally, we now show a similar weak interaction of p115-RhoGEF(EED) with $\alpha_{12}$ QL (Fig. 5A). Surprisingly, however, whereas $\alpha_{13} \mathrm{QL}$ efficiently recruits p115-RhoGEF(EED) to the PM and the membrane fraction, $\alpha_{12} \mathrm{QL}$ fails to do so (Fig. 6). Taken together, the results show clear differences in the mechanisms of $\alpha_{13}$ versus $\alpha_{12}$ to mediate the PM recruitment of p115-RhoGEF and support the idea that PM recruitment mediated by $\alpha_{12}$ relies predominantly on interactions with the RGS domain, but PM recruitment mediated by $\alpha_{13}$ utilizes multiple mechanisms. 
Although these studies are the first to describe differences between $\alpha_{13}$ and $\alpha_{12}$ in terms of PM recruitment of an RGS-RhoGEF, some important differences in interaction have been noted. For example, $\alpha_{13}$ is a better substrate than is $\alpha_{12}$ for the RGS domain-mediated GAP activity of p115-RhoGEF or LARG [6,29]. Moreover, activated $\alpha_{13}$ stimulates the RhoGEF activity of p115-RhoGEF or LARG in assays in vitro, but activated $\alpha_{12}$ is unable to do so [6,34]. In fact, $\alpha_{12}$ can inhibit the ability of $\alpha_{13}$ to activate $\mathrm{p} 115$-RhoGEF in vitro [34]. It has thus been suggested that $\alpha_{13}$ activates an RGS-RhoGEF by interacting with two distinct surfaces, i.e., the RGS domain and the DH/PH domain, while $\alpha_{12}$ would prevent $\alpha_{13}$ interaction by binding only to the RGS domain $[4,6]$. Our results, examining the requirements for PM recruitment of p115-RhoGEF by $\alpha_{13}$ or $\alpha_{12}$, are consistent with this model. However, the exact role in cells for $\alpha_{13}$ versus $\alpha_{12}$ in regulating p115-RhoGEF remains to be established, and whether $\alpha_{12}$ activates or inhibits p115-RhoGEF in vivo is still an open question. So far, there exists no clear evidence to prove or refute the proposal that $\alpha_{12}$ indeed activates Rho via p115-RhoGEF. Interesting, $\alpha_{12}$ is unable to activate LARG in vitro unless LARG has undergone tyrosine phosphorylation; it is possible that $\mathrm{p} 115-\mathrm{RhoGEF}$ also undergoes a covalent modification in cells that confers it with the ability to then be positively regulated by $\alpha_{12}$.

\section{CONCLUSION}

In summary, the results presented here not only indicate the importance of regulated PM localization for $\mathrm{p} 115-\mathrm{RhoGEF}$ function, but also describe an unexpected role for Rho in the PM recruitment of p115-RhoGEF and unexpected differences in the mechanisms used by $\alpha_{13}$ and $\alpha_{12}$ to promoted the PM recruitment of p115-RhoGEF.

\section{Supplementary Material}

Refer to Web version on PubMed Central for supplementary material.

\section{Acknowledgements}

This work was supported by NIH grant (to K. K.), NIH grant GM62884 (to P. W.) and a fellowship from the American Heart Association Pennsylvania-Delaware Affiliate (to R. B.). We thank Katarzyna Urbanska for her assistance in performing the TUNEL assay.

\section{References}

1. Cabrera-Vera TM, Vanhauwe J, Thomas TO, Medkova M, Preininger A, Mazzoni MR, Hamm HE. Endocr Rev 2003;24(6):765-781. [PubMed: 14671004]

2. Kelly P, Casey PJ, Meigs TE. Biochemistry 2007;46(23):6677-6687. [PubMed: 17503779]

3. Rossman KL, Der CJ, Sondek J. Nat Rev Mol Cell Biol 2005;6(2):167-180. [PubMed: 15688002]

4. Sternweis PC, Carter AM, Chen Z, Danesh SM, Hsiung YF, Singer WD. Adv Protein Chem 2007;74:189-228. [PubMed: 17854659]

5. Wells CD, Liu MY, Jackson M, Gutowski S, Sternweis PM, Rothstein JD, Kozasa T, Sternweis PC. J Biol Chem 2002;277(2):1174-1181. [PubMed: 11698392]

6. Kreutz B, Hajicek N, Yau DM, Nakamura S, Kozasa T. Cell Signal 2007;19(8):1681-1689. [PubMed: 17449226]

7. Suzuki N, Nakamura S, Mano H, Kozasa T. Proc Natl Acad Sci U S A 2003;100(2):733-738. [PubMed: 12515866]

8. Holinstat M, Mehta D, Kozasa T, Minshall RD, Malik AB. J Biol Chem 2003;278(31):28793-28798. [PubMed: 12754211]

9. Wells CD, Gutowski S, Bollag G, Sternweis PC. J Biol Chem 2001;276(31):28897-28905. [PubMed: 11384980]

10. Grabocka E, Wedegaertner PB. Mol Pharmacol 2007;72(4):993-1002. [PubMed: 17609419] 
11. Grabocka E, Wedegaertner PB. Oncogene 2005;24(13):2155-2165. [PubMed: 15735747]

12. Bhattacharyya R, Wedegaertner PB. Biochem J 2003;371(Pt 3):709-720. [PubMed: 12534370]

13. Bhattacharyya R, Wedegaertner PB. FEBS Lett 2003;540(13):211-216. [PubMed: 12681510]

14. Bhattacharyya R, Wedegaertner PB. Journal of Biological Chemistry 2000;275(20):14992-14999. [PubMed: 10747909]

15. Meyer BH, Freuler F, Guerini D, Siehler S. J Cell Biochem 2008;104(5):1660-1670. [PubMed: 18320579]

16. Park SY, Li H, Avraham S. Cell Signal 2007;19(2):289-300. [PubMed: 16945503]

17. Aoki J, Katoh H, Yasui H, Yamaguchi Y, Nakamura K, Hasegawa H, Ichikawa A, Negishi M. Biochem J 1999;340(Pt 2):365-369. [PubMed: 10333476]

18. Nusser N, Gosmanova E, Makarova N, Fujiwara Y, Yang L, Guo F, Luo Y, Zheng Y, Tigyi G. Cell Signal 2006;18(5):704-714. [PubMed: 16109481]

19. Jalink K, van Corven EJ, Hengeveld T, Morii N, Narumiya S, Moolenaar WH. J Cell Biol 1994;126 (3):801-810. [PubMed: 8045941]

20. Tigyi G, Fischer DJ, Sebok A, Yang C, Dyer DL, Miledi R. J Neurochem 1996;66(2):537-548. [PubMed: 8592123]

21. Katoh H, Aoki J, Yamaguchi Y, Kitano Y, Ichikawa A, Negishi M. Journal of Biological Chemistry 1998;273(44):28700-28707. [PubMed: 9786865]

22. Aktories K, Wilde C, Vogelsgesang M. Rev Physiol Biochem Pharmacol 2004;152:1-22. [PubMed: 15372308]

23. Choy E, Chiu VK, Silletti J, Feoktistov M, Morimoto T, Michaelson D, Ivanov IE, Philips MR. Cell 1999;98(1):69-80. [PubMed: 10412982]

24. Apolloni A, Prior IA, Lindsay M, Parton RG, Hancock JF. Mol Cell Biol 2000;20(7):2475-2487. [PubMed: 10713171]

25. Wang JY, Grabacka M, Marcinkiewicz C, Staniszewska I, Peruzzi F, Khalili K, Amini S, Reiss K. J Neurosci Res 2006;83(1):7-18. [PubMed: 16307448]

26. Royuela M, Rodriguez-Berriguete G, Fraile B, Paniagua R. Histol Histopathol 2008;23(10):1279_ 1290. [PubMed: 18712680]

27. Copeland JW, Treisman R. Mol Biol Cell 2002;13(11):4088-4099. [PubMed: 12429848]

28. Deroanne C, Vouret-Craviari V, Wang B, Pouyssegur J. J Cell Sci 2003;116(Pt 7):1367-1376. [PubMed: 12615978]

29. Kozasa T, Jiang X, Hart MJ, Sternweis PM, Singer WD, Gilman AG, Bollag G, Sternweis PC. Science 1998;280(5372):2109-2111. [PubMed: 9641915]

30. Kreutz B, Yau DM, Nance MR, Tanabe S, Tesmer JJ, Kozasa T. Biochemistry 2006;45(1):167-174. [PubMed: 16388592]

31. Chen Z, Singer WD, Sternweis PC, Sprang SR. Nat Struct Mol Biol 2005;12(2):191-197. [PubMed: 15665872]

32. Chen Z, Singer WD, Wells CD, Sprang SR, Sternweis PC. J Biol Chem 2003;278(11):9912-9919. [PubMed: 12525488]

33. Kitzing TM, Sahadevan AS, Brandt DT, Knieling H, Hannemann S, Fackler OT, Grosshans J, Grosse R. Genes Dev 2007;21(12):1478-1483. [PubMed: 17575049]

34. Hart MJ, Jiang X, Kozasa T, Roscoe W, Singer WD, Gilman AG, Sternweis PC, Bollag G. Science 1998;280(5372):2112-2114. [PubMed: 9641916]

\section{Abbreviations used}

\section{G protein}

guanine nucleotide-binding protein

PM

plasma membrane

\section{HEK293 cells}


human embryonic kidney cells

HA

hemagglutinin

PAGE

polyacrylamide gel electrophoresis

PVDF

polyvinylidene difluoride

RGS

regulator of $\mathrm{G}$ protein signaling

GEF

guanine-nucleotide exchange factor

NGF

nerve growth factor 
A.

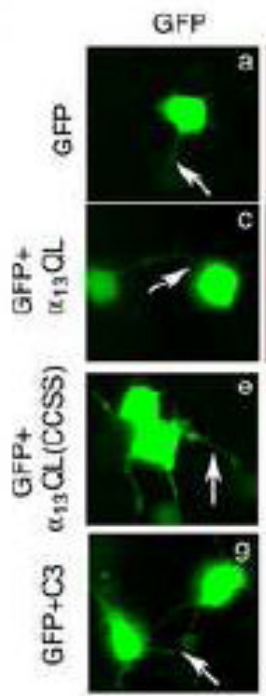

anti-HA
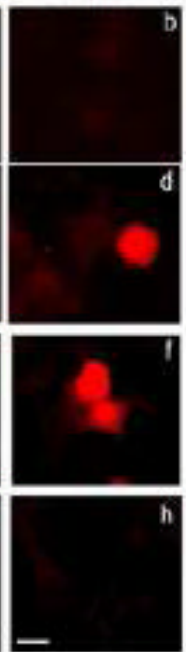
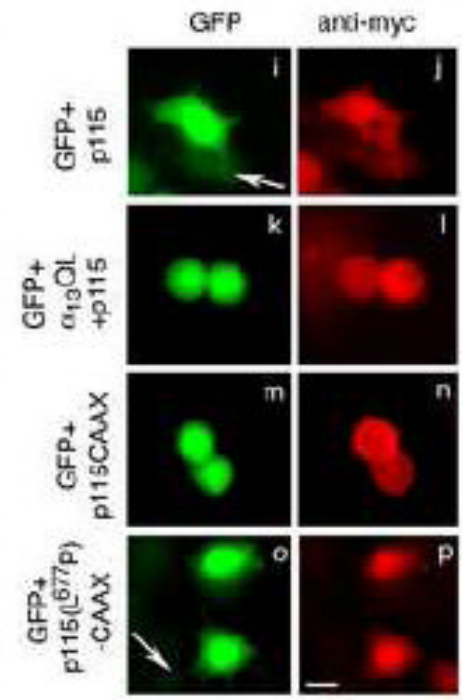

B.
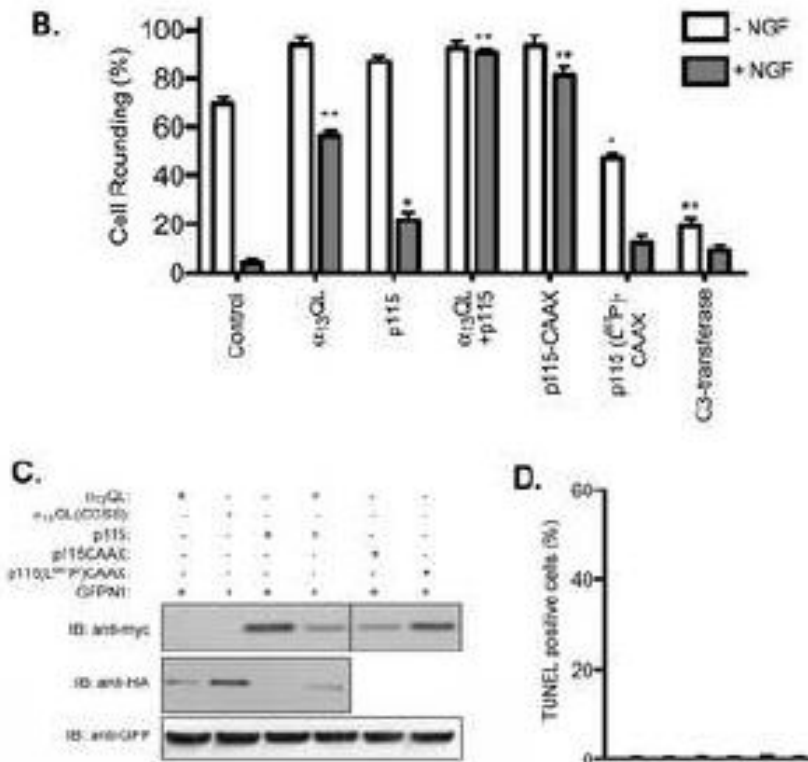

D.

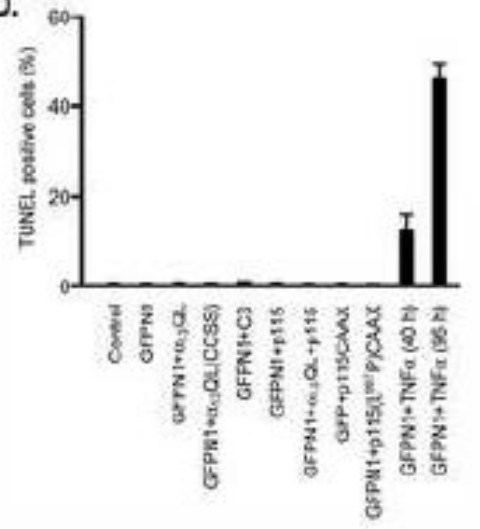

Figure 1. Rho-dependent neurite retraction in PC12 cells by overexpression of $\alpha_{13} \mathrm{QL}, \mathrm{p} 115$ RhoGEF and their mutants

A. PC12 cells were transfected with plasmids encoding GFP along with vector (a and b), HA$\alpha_{13} \mathrm{QL}$ (c and d), HA- $\alpha_{13} \mathrm{QL}(\mathrm{CCSS}$ ) (e and f), myc-tagged C3-transferase ( $\mathrm{g}$ and $\mathrm{h}$ ), myctagged p115-RhoGEF ( $i$ and j), myc-p115-RhoGEF and HA- $\alpha_{13}$ QL (k and l), myc-p115RhoGEF-CAAX ( $m$ and n), and myc-p115(L677P)-CAAX (o and p). 24 h post-transfection cells were transferred into serum free medium containing $100 \mathrm{ng} / \mathrm{ml}$ NGF. $16 \mathrm{~h}$ post-NGFtreatment cells were fixed and labeled with anti-HA antibody (anti-HA) or anti-myc antibody (anti-myc) followed by Texas Red-conjugated anti-mouse secondary antibody. The neuronal morphology was detected by observing the distribution of GFP in the cells co-expressing the 
indicated proteins. Representative images are presented. The arrows indicate neurite formation. Note that the GFP images are purposely overexposed so that the neurites are visible. Bar, 10 $\mu \mathrm{m}$. $\boldsymbol{B}$. A quantitative analysis of cell rounding is represented by plotting percent (\%) of round cells calculated in each transfection experiment, as described in "Materials and Methods." The data are average +/- S.E. of three separate experiments. Statistical significance (student's ttest $)$, indicated by $*(p<0.05)$ and $* *(p<0.001)$, is shown and compares untreated samples (-NGF) to control and NGF-treated (+NGF) to control. $\boldsymbol{C}$. Total cell lysates from the indicated transfections of PC12 cells were immunoblotted with anti-myc, anti-HA and anti-GFP antibodies to determine expression levels of GFP, p115-RhoGEF, $\alpha_{13} \mathrm{QL}$ and their mutants. D. $40 \mathrm{~h}$ post-transfection, differentiating PC12 cells were subjected to TUNEL assay as described in the "Materials and Methods" section. The data is a representative experiment performed in triplicate. 

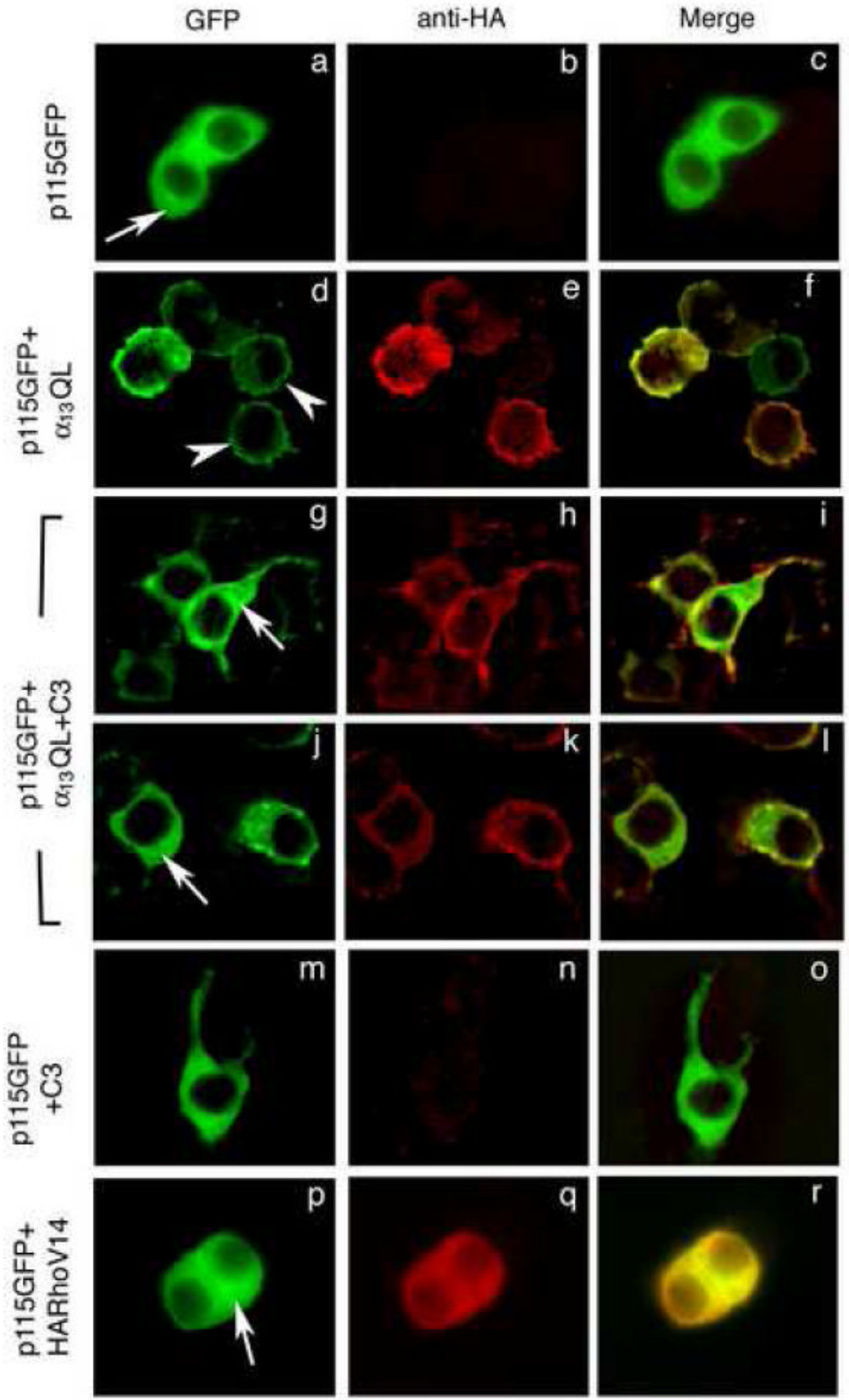

Figure 2. Inhibition of Rho prevents $\alpha_{13} Q L$-induced PM translocation of p115-RhoGEF in PC12 cells

PC12 cells were transiently transfected with plasmids encoding GFP-tagged p115-RhoGEF (p115GFP), C3-transferase (C3), HA-tagged RhoV14 (HARhoV14) or $\alpha_{13} \mathrm{QL}\left(\alpha_{13} \mathrm{QL}\right)$, in various combinations as indicated. $16 \mathrm{~h}$ after transfection, cells were fixed and stained with anti-HA monoclonal antibody (b, e, h, k, n, and q) followed by anti-mouse secondary antibody conjugated to Texas Red to detect $\alpha_{13} \mathrm{QL}$ or RhoV14. p115-RhoGEF was detected due to the intrinsic fluorescence of the fused GFP (a, d, g, j, m, and p). Arrows indicate cytoplasmic staining, and arrowheads indicate plasma membrane staining. Representative images were recorded by immunofluorescence microscopy. 
A.

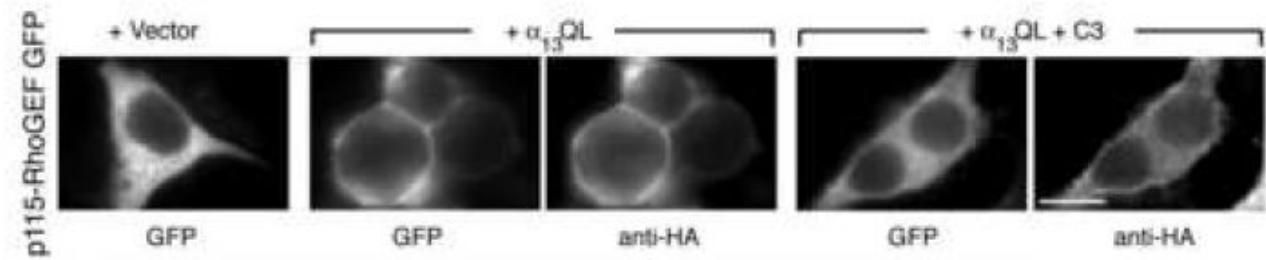

B.

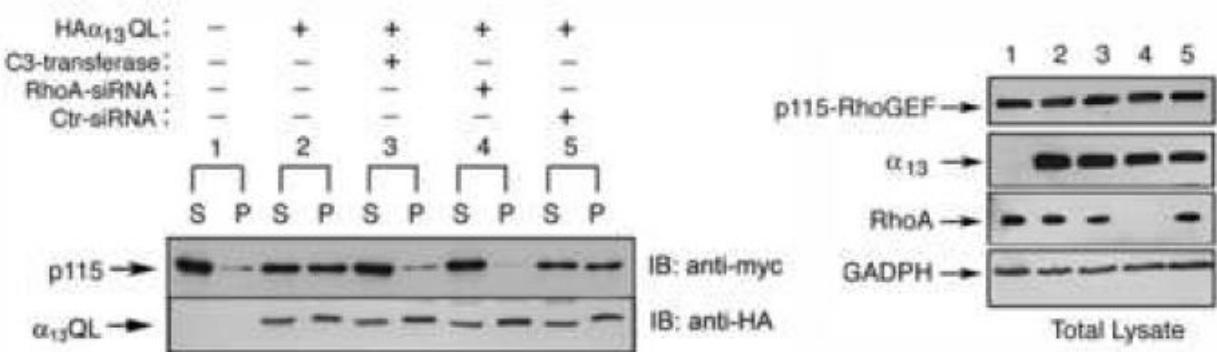

C.

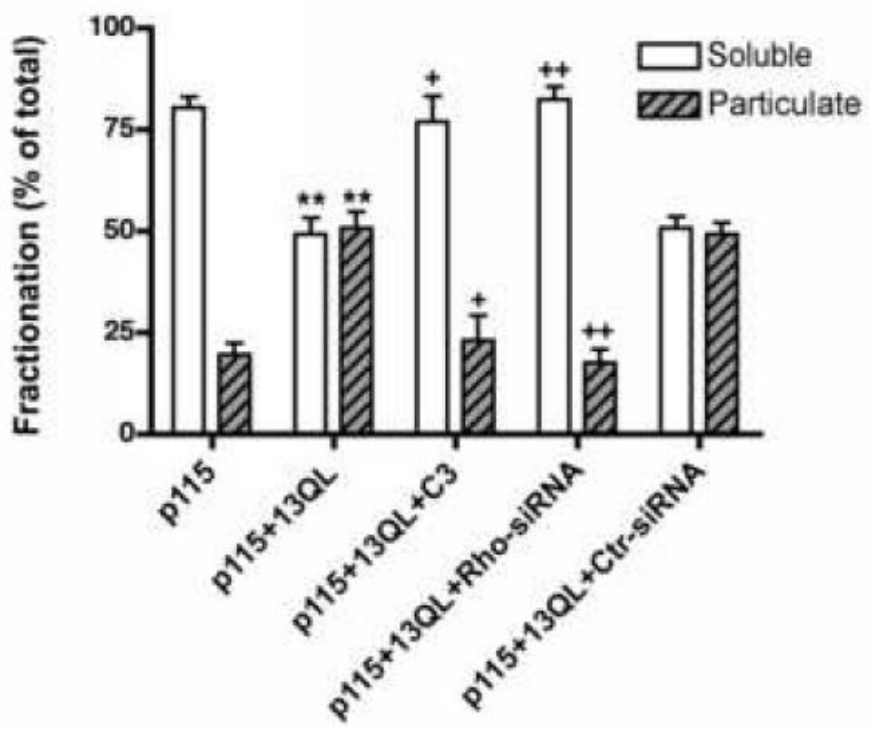

Figure 3. Inhibition or depletion of Rho prevents $\alpha_{13} \mathrm{QL}$-induced PM recruitment of p115-RhoGEF in HEK293 cells

$\boldsymbol{A}$. HEK293 cells were transfected with the expression vector for p115-RhoGEF-GFP along with empty vector, an expression plasmid for HA-tagged $\alpha_{13} \mathrm{QL}$, or expression plasmids for both HA-tagged and $\alpha_{13}$ QL myc-tagged C3-transferase (C3), as indicated. $24 \mathrm{~h}$ posttransfection, cells were fixed and subjected to immunofluorescence microscopy as described in the "Materials and Methods." $\boldsymbol{B}$. HEK293 cells were transfected with plasmids encoding myc-tagged $\mathrm{p} 115$-RhoGEF along with expression plasmids for HA-tagged $\alpha_{13} \mathrm{QL}$, myc-tagged C3-transferase, siRNA specific for Rho silencing (RhoA-siRNA) or non-specific siRNA (CtrsiRNA), as indicated (left panel). Cells were lysed and fractionated into soluble (S) and 
particulate (P) fractions, as described in the "Materials and Methods." The fractions were immunoblotted (IB) with anti-myc antibody to detect p115-RhoGEF and anti-HA antibody to detect $\alpha_{13} \mathrm{QL}$. Total cell lysates were immunoblotted (right panel) with anti-myc antibody and anti-HA antibody to show equivalent expression of p115-RhoGEF and $\alpha_{13} \mathrm{QL}$, respectively. In addition, effective knockdown of endogenous Rho was detected by probing cell lysates with anti-Rho antibody, and immunoblotting with an anti-GADPH antibody served as a loading control. The lane numbering in the right panel indicates that the cell lysate corresponds to the transfection indicated in the left panel. $\boldsymbol{C}$. Data from three independent fractionation experiments of p115-RhoGEF were quantitated (mean + /- S.E.). Statistical significance (student's t-test), indicated by ** $(\mathrm{p}<0.005)$, compares $\mathrm{p} 115-\mathrm{RhoGEF}$ alone to $\mathrm{p} 115-\mathrm{RhoGEF}$ in the presence of co-expressed $\alpha_{13} \mathrm{QL} ;+(\mathrm{p}<0.05)$ and $++(\mathrm{p}<0.005)$ indicates significance in comparison to $\mathrm{p} 115-\mathrm{RhoGEF}+\alpha_{13} \mathrm{QL}$. Soluble fractions (white bars) are compared to other soluble fractions, while particulate fractions (black bars) are compared to other particulate fractions. 
A.

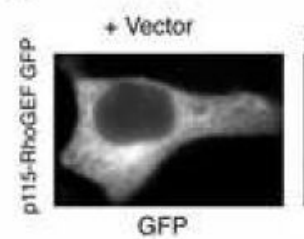

GFP

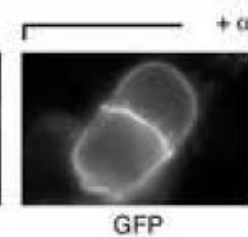

GFP

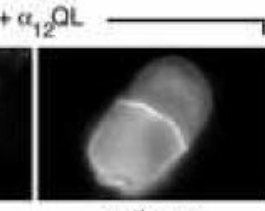

anti- $\alpha_{12}$

B.

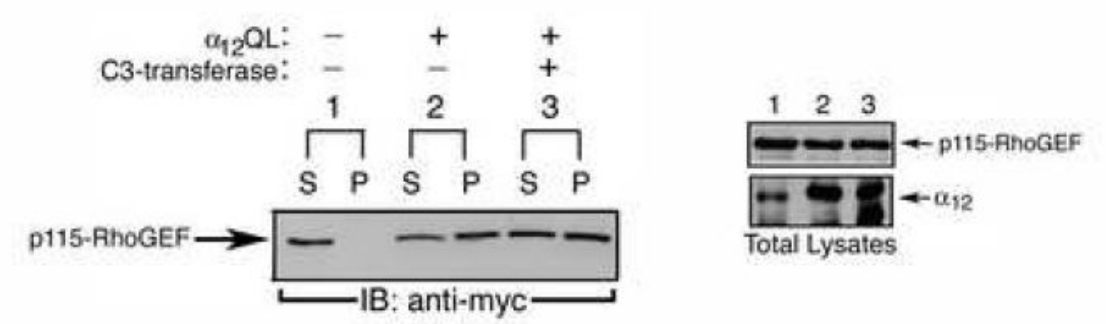

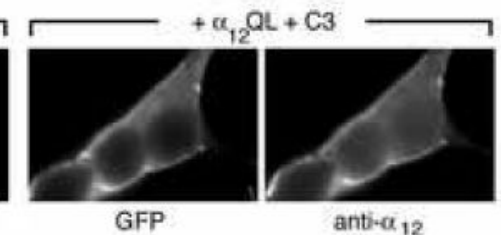

anti- $\alpha_{12}$

Figure 4. $\alpha_{12} \mathrm{QL}$-induced PM recruitment of p115-RhoGEF is refractory to inhibition of Rho $\boldsymbol{A}$. HEK293 cells were transfected with the expression vector for $\mathrm{p} 115-\mathrm{RhoGEF}-\mathrm{GFP}$ along with empty vector, an expression plasmid for $\alpha_{12} \mathrm{QL}$, or expression plasmids for both $\alpha_{12} \mathrm{QL}$ and myc-tagged $\mathrm{C} 3$-transferase (C3), as indicated. $24 \mathrm{~h}$ post-transfection, cells were fixed and subjected to immunofluorescence microscopy as described in the "Materials and Methods." $\boldsymbol{B}$. HEK293 cells were transfected with plasmids encoding myc-tagged p115-RhoGEF along with expression plasmids for $\alpha_{12} \mathrm{QL}$ and/or myc-tagged C3-transferase, as indicated (left panel). Cells were lysed and fractionated into soluble (S) and particulate $(\mathrm{P})$ fractions, as described in the "Materials and Methods." The fractions were immunoblotted (IB) with antimyc antibody to detect p115-RhoGEF. Total cell lysates were immunoblotted (right panel) with anti-myc antibody and anti- $\alpha_{12}$ antibody to show expression of p115-RhoGEF and $\alpha_{12} \mathrm{QL}$, respectively. The lane numbering in the right panel indicates that the cell lysate corresponds to the transfection indicated in the left panel. 

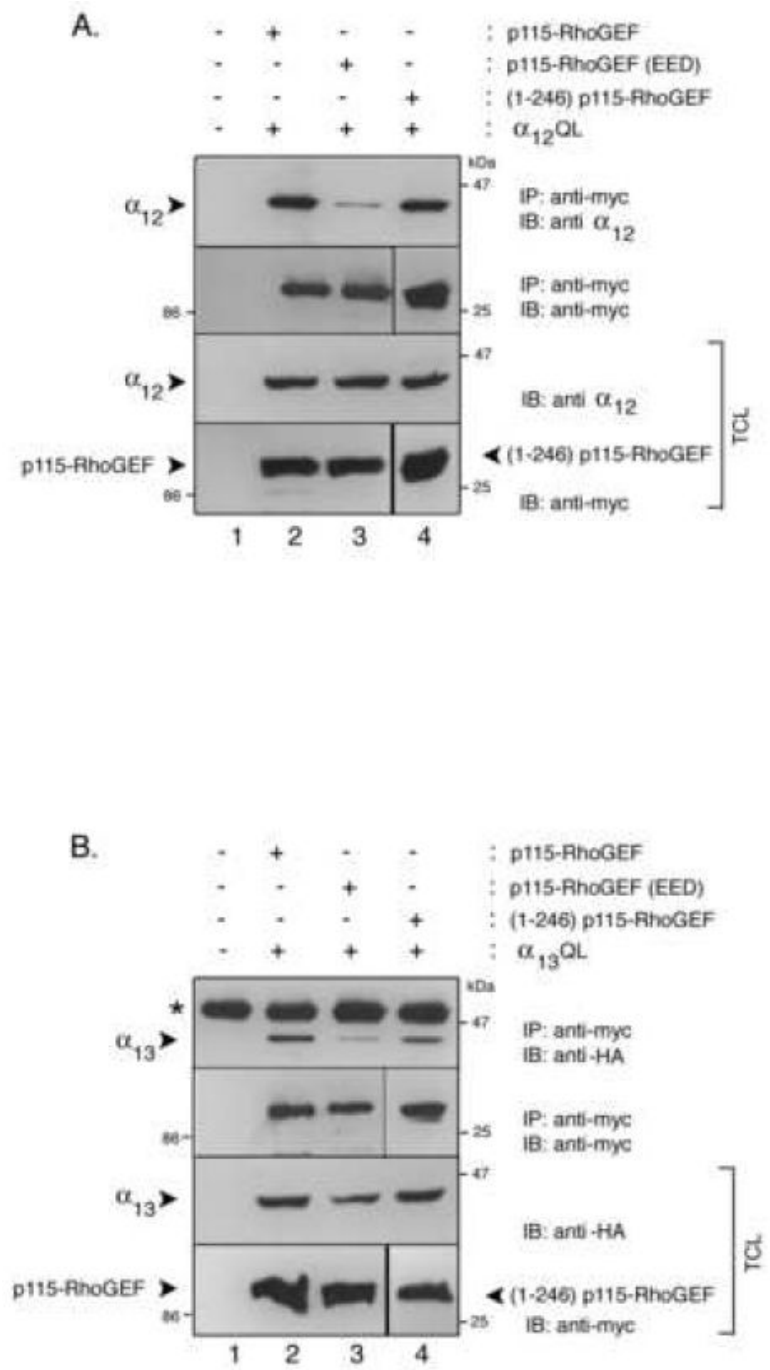

Figure 5. Co-immunoprecipitation of $\alpha_{12} \mathrm{QL}$ and $\alpha_{13} \mathrm{QL}$ with p115-RhoGEF and p115-RhoGEF RGS domain mutants

$\boldsymbol{A}$. HEK293 cells were transfected with empty vector (lane 1), expression plasmids for $\alpha_{12} \mathrm{QL}$ and p1 15-RhoGEF (lane 2), expression plasmids for $\alpha_{12} \mathrm{QL}$ and p115-RhoGEF(EED) (lane 3), or expression plasmids for $\alpha_{12} \mathrm{QL}$ and (1-246)p115-RhoGEF (lane 4). Cells were lysed and the lysates were subjected to immunoprecipitation (IP) by monoclonal anti-myc antibody to precipitate p115-RhoGEF and its mutants. The immunoprecipitates were immunoblotted (IB) with polyclonal anti- $\alpha_{12}$ antibody (upper panel) and anti-myc antibody (second panel). The corresponding total cell lysates were immunoblotted and probed with anti-myc or anti- $\alpha_{12}$ antibodies to show expression of $\mathrm{p} 115$-RhoGEF and its mutants (lower panel), and $\alpha_{12} \mathrm{QL}$ (third panel). B. HEK293 cells were transfected with empty vector (lane 1), expression plasmids for $\alpha_{13} \mathrm{QL}$ and p115-RhoGEF (lane 2), expression plasmids for $\alpha_{13} \mathrm{QL}$ and p115RhoGEF(EED) (lane 3), or expression plasmids for $\alpha_{13} \mathrm{QL}$ and (1-246)p115-RhoGEF (lane 4). Cells were lysed and the lysates were subjected to immunoprecipitation (IP) by monoclonal anti-myc antibody to precipitate $\mathrm{p} 115-\mathrm{RhoGEF}$ and its mutants. The immunoprecipitates were immunoblotted (IB) with polyclonal anti-HA antibody (upper panel) to detect $\alpha_{13} \mathrm{QL}$ and antimyc antibody (second panel). The corresponding total cell lysates were immunoblotted and probed with anti-myc or anti-HA antibodies to show expression of p115-RhoGEF and its mutants (lower panel), and $\alpha_{13} \mathrm{QL}$ (third panel). 
A.
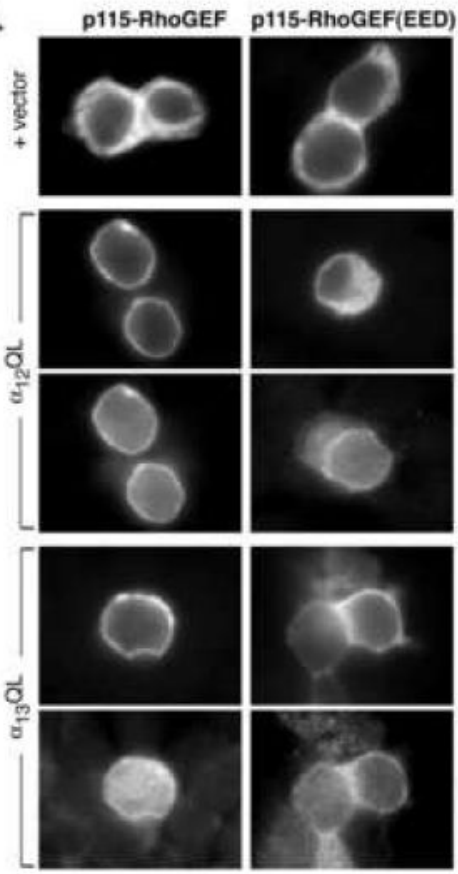
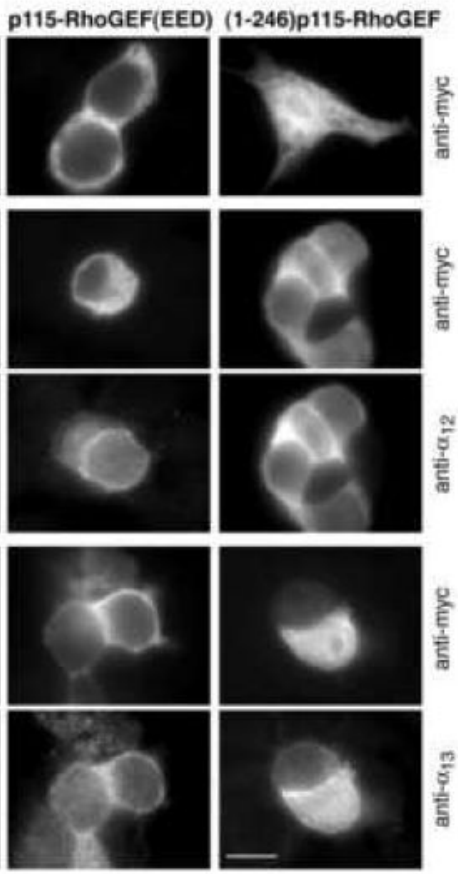

B.
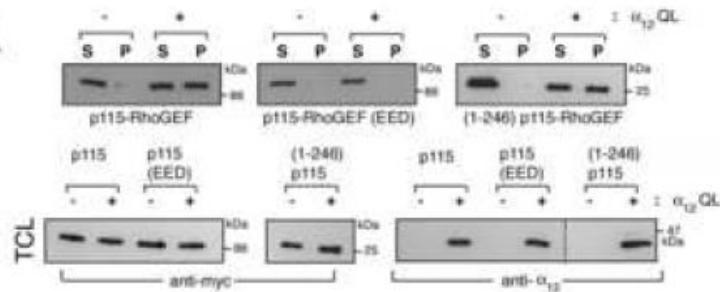

C.
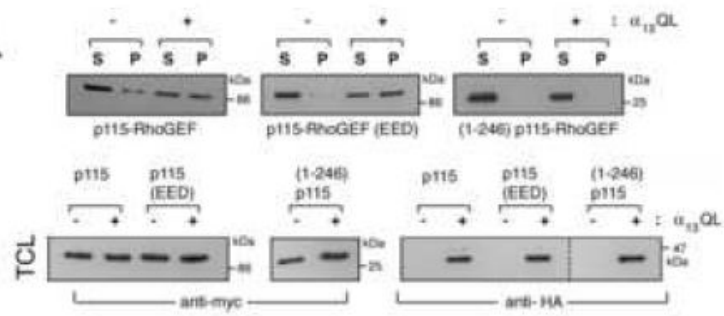

Figure 6. Differential $\alpha_{12} \mathrm{QL}$ - and $\alpha_{13} \mathrm{QL}$-mediated PM recruitment of p115-RhoGEF RGS domain mutants

$\boldsymbol{A}$. HEK293 cells were transfected with either p115-RhoGEF (left column), p115-RhoGEF (EED) (middle column), or (1-246)p115-RhoGEF (right column). Each p115-RhoGEF wt or mutant was co-transfected with empty vector (upper row), $\alpha_{12} \mathrm{QL}$ (rows 2 and 3), or $\alpha_{13} \mathrm{QL}$ (rows 4 and 5). $24 \mathrm{~h}$ post-transfection, cells were fixed and subjected to immunofluorescence microscopy as described in the "Materials and Methods," using the indicated antibodies. $\boldsymbol{B}$. HEK293 cells were transfected with either p115-RhoGEF, p115-RhoGEF(EED), or (1-246) p115-RhoGEF. Each p115-RhoGEF wt or mutant was co-transfected with empty vector or $\alpha_{12} \mathrm{QL}$, as indicated. Cells were lysed and fractionated into soluble (S) and particulate (P) fractions, as described in the "Materials and Methods." The fractions were immunoblotted (IB) with anti-myc antibody to detect p115-RhoGEF (upper panels). Total cell lysates (TCL) were immunoblotted with anti-myc antibody and anti- $\alpha_{12}$ antibody to show expression of p115- 
RhoGEF and $\alpha_{12}$ QL, respectively (lower panels). C. HEK293 cells were transfected with either p115-RhoGEF, p115-RhoGEF(EED), or (1-246)p115-RhoGEF. Each p115-RhoGEF wt or mutant was co-transfected with empty vector or $\alpha_{13} \mathrm{QL}$, as indicated. Cells were lysed and fractionated into soluble (S) and particulate $(\mathrm{P})$ fractions, as described in the "Materials and Methods." The fractions were immunoblotted (IB) with anti-myc antibody to detect p115-

RhoGEF (upper panels). Total cell lysates were immunoblotted with anti-myc antibody and anti-HA antibody to show expression of p115-RhoGEF and $\alpha_{13} \mathrm{QL}$, respectively (lower panels). The dashed lines (lower right panels in $\mathrm{B}$ and $\mathrm{C}$ ) indicate that the samples are from the same gel; intervening lanes that were irrelevant to the figure were simply removed. 